\title{
Study of antiepileptic drug fosphenytoin; a prodrug of phenytoin prodrug fosphenytoin
}

\begin{abstract}
Fosphenytoin is a phenytoin prodrug that has been introduced to overcome some of the problems and limitations associated with parenteral phenytoin sodium administration. Fosphenytoin is a phosphate ester prodrug that is converted to phenytoin in vivo by peripheral esterases. Fosphenytoin has several advantages and disadvantages that should be considered when selecting its use in place of parenteral phenytoin. Advantages with Fosphenytoin include better tolerability, improved safety, better stability, ability for I.M administration, and faster infusion rates. Disadvantages with Fosphenytoin include rate and dose related paresthesias and pruritus, delayed decreases in blood pressure, the potential for therapeutic drug monitoring errors, and higher drug acquisition costs.
\end{abstract}

Volume 2 Issue 2 - 2018

\author{
Mohammad Asif \\ Department of Pharmacy, India
}

Correspondence: Mohammad Asif, Department of Pharmacy, India,Tel 052-4262478, Email aasif32I@gmail.com

Received: November 18, 2017 | Published: March 22, 2018

Keywords: fosphenytoin, phenytoin, antiepileptic, prodrug

\section{Introduction}

Fosphenytoin, the disodium phosphate ester of 3hydroxymethyl-5,5-diphenylhydantoin (FPHT/ACC 9653/Cerebyx), phosphate ester pro-drug of phenytoin, 5,5-diphenylhydantoin (PHT/ Dilantin), was developed to overcome complications associated with parenteral PHT administration in treatment of seizures, short-term prophylaxis and treatment of repetitive or prolonged seizures and status epilepticus (SE). Effect of pharmacokinetics, dynamics, clinical efficacy and tolerability of FPHT in children and adults studied. The intravenous (i.m.) FPHT has a similar adverse effect profile than PHT. ${ }^{1}$ The effect of FPHT, single or adjunctive antiepileptic (AE) in SE, intraperitoneal (i.p.) therapeutically $5 \mathrm{~min}$ after seizure onset, only the highest FPHT dose $(180 \mathrm{mg} / \mathrm{kg})$ was capable of terminating seizure activity in $50 \%$ of the tested animals. FPHT $(18-180 \mathrm{mg} /$ $\mathrm{kg}$ ) was administered as a pretreatment, i.p., seizures were blocked in a dose-dependent fashion. Combinations of diazepam and FPHT were also effective. FPHT $(18-56 \mathrm{mg} / \mathrm{kg})$, given in conjunction with a diazepam $(4.8 \mathrm{mg} / \mathrm{kg}$, i.m.) $5 \mathrm{~min}$ after seizure onset, enhanced the AE effect of diazepam. When FPHT (18 or $32 \mathrm{mg} / \mathrm{kg}$, i.p.) was given as a pretreatment and diazepam was given 5 min after seizure onset, the $32 \mathrm{mg} / \mathrm{kg}$ dose of FPHT significantly reduced the time for seizure control. The FPHT, either alone or in combination with diazepam, has little or no therapeutic AE effects for nerve agent-induced SE. ${ }^{2}$ Single intramuscular (I.M) injection of FPHT prevents seizures. FPHT (20 PHT equivalents $/ \mathrm{kg}$ ) examined the prevalence and frequency of clinical seizures. A single i.m injection of FPHT (20 PHT equivalents/ $\mathrm{kg}$ ) does not prevent seizures or neurologic deficits in childhood acute nontraumatic coma. ${ }^{3}$ Oral PHT, I.V. PHT, and I.V FPHT are all commonly used for loading PHT in the emergency department. ${ }^{4}$ PHT is a first-line drug in the treatment of SE. The parenteral PHT formulation is associated with administration difficulties and therefore FPHT, has been developed. PHT and FPHT were administered by i.v. infusion and blood, cerebrospinal fluid (CSF) and microdialysate samples. The pharmacokinetic parameters in plasma of PHT after PHT and FPHT (30 and $60 \mathrm{mg} / \mathrm{kg}$ ) administration were indistinguishable. The PHT plasma free fraction was $0.25-0.31$ and $0.26-0.31$ for PHT and FPHT, respectively. Cmax values increased dose-dependently and were independent of whether PHT or FPHT was administered.
The kinetic profiles of PHT for the frontal cortex and hippocampus were indistinguishable suggesting that PHT distribution in the brain is not brain region specific. Thus, overall, the central and peripheral kinetics of PHT are indistinguishable after PHT and FPHT. ${ }^{5}$ The SE is common in children with severe falciparum malaria and is associated with poor outcome. PHT is often used to control SE, but its watersoluble prodrug, FPHT, may be more useful as it is easier to administer. The pharmacokinetics and clinical effects of PHT and FPHT sodium in children with severe falciparum malaria and SE, PHT and FPHT administration at the currently recommended doses achieve plasma unbound PHT concentrations within the therapeutic range with few cardiovascular effects. Administration of FPHT i.v. or i.m. offers a convenient alternative to i.m. PHT. ${ }^{6}$ Several AEDs are therapeutic in mania and since acute mania requires rapid and intensive treatment, i.v. high-dose PHT might be acutely antimanic. A prodrug of PHT, FPHT, which has few cardiac or local vein side effects, was also used and no antimanic effects were observed. The i.v. FPHT at doses that are effective in SE appears to be ineffective in acute mania. ${ }^{7}$

FPHT is a phosphate ester prodrug, an alternative to i.v. PHT for acute treatment of seizures. Advantages include more convenient and rapid i.v. administration, availability for i.m. injection, and low potential for adverse local reactions at injection sites. Drawbacks include the occurrence of transient paraesthesias and pruritus at rapid infusion rates, and cost. FPHT is highly bound (93-98\%) to plasma proteins. Saturable binding at higher plasma concentrations accounts for an increase in its distribution volume and clearance with increasing dose and infusion rate. FPHT is entirely eliminated through metabolism to PHT by blood and tissue phosphatases. The bioavailability of the derived PHT relative to i.v. PHT is approximately $100 \%$ following i.v. or i.m. administration. The halflife for conversion of FPHT to PHT ranges from 7-15 minutes. Faster i.v. infusion rates and competitive displacement of derived PHT from plasma protein binding sites by FPHT compensate for the expected conversion-related delay in appearance of PHT in the plasma. Unbound PHT plasma concentrations achieved with i.v. FPHT loading doses of $100-150$ or $50-100 \mathrm{mg}$ PHT sodium equivalents/ min are comparable, and achieved at similar times, to those with equimolar doses of i.v. PHT at 50 or $20-40 \mathrm{mg} / \mathrm{min}$, respectively. 
The rapid achievement of effective concentrations permits the use of FPHT in emergency situations. Plasma concentration profiles for FPHT and total and unbound PHT in infants and children closely approximate those in adults following i.v. or i.m. FPHT at comparable doses and infusion rates. Earlier and higher unbound PHT plasma concentrations, and thus an increase in systemic adverse effects, may occur following i.v. FPHT loading doses in patients with a decreased ability to bind FPHT and PHT (renal or hepatic disease, hypo-albuminaemia, the elderly). The potential exists for clinically significant interactions when FPHT is co-administered with other highly protein bound drugs. The pharmacokinetic properties of FPHT permit the drug to serve as a well tolerated and effective alternative to parenteral PHT in the emergency and non-emergency management of acute seizures in children and adults. ${ }^{8}$ FPHT is metabolized by phosphatases to yield the AED PHT plus inorganic phosphate. Thus, FPHT can theoretically alter the electrocardiogram by 2 mechanisms: the direct effects of PHT on cardiac conduction and on phosphate binding of calcium, which could indirectly alter cardiac conduction as a result of hypocalcemia. The patient was normocalcemic before drug infusion. FPHT produced electro-cardiographic changes (prolongation of the ST segment and the QT interval and merging of the $\mathrm{T}$ and $\mathrm{P}$ waves) consistent with hypocalcemia, and these changes were associated with new-onset reductions in both total and ionized serum calcium concentrations. Plasma PHT concentrations were within the therapeutic range during the electro cardiographic changes and the patient's blood pressure was stable. These findings showed as FPHT-related electrocardiographic changes likely attributable to inorganic phosphate-induced hypocalcemia. ${ }^{9}$

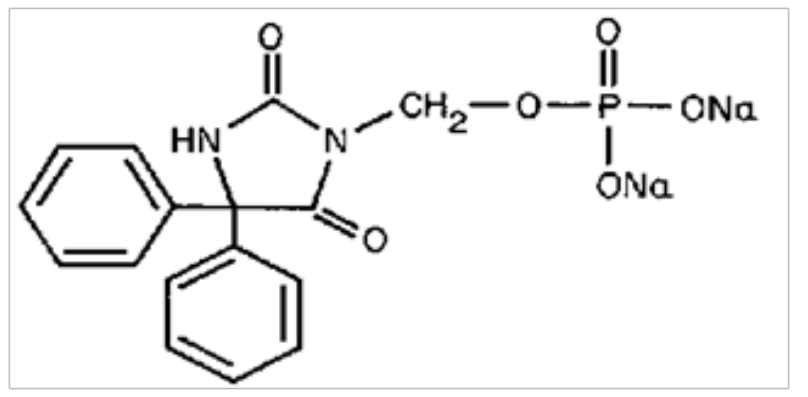

Figure I: Structure of Fosphenytoin.

The compare plasma PHT concentration versus time profiles following i.v. and i.m. administration of FPHT sodium with those obtained administration of standard PHT sodium injection in the rabbit. Plasma was separated by centrifugation and FPHT, total and free plasma PHT concentrations were measured. Following i.v. administration of FPHT sodium plasma PHT concentrations were similar to those obtained following i.v. administration of an equivalent dose of PHT sodium. Mean peak plasma PHT concentrations (Cmax) was $158 \%$ higher following i.m. administration of FPHT sodium compared to i.m. administration of PHT sodium. There was no significant difference in AUC $(0-180)$ between FPHT and PHT-treated rabbits following i.v. administration. There was also no significant difference in the mean times to achieve peak plasma PHT concentrations (Tmax) between FPHT and PHT-treated rabbits following i.m. administration. Mean plasma albumin concentrations were comparable in both groups of animals. FPHT was rapidly converted to PHT both after i.v. and i.m. administration, with plasma FPHT concentrations declining rapidly to undetectable levels within 10 min following administration via either route. The results confirm the rapid and complete hydrolysis of FPHT to PHT in vivo, and the potential of the i.m. route for administration of FPHT delivering PHT in clinical settings where i.v. administration may not be feasible. ${ }^{10}$ The conversion of FPHT to PHT was slow in sera with normal alkaline phosphatase activities. The conversion was rapid in sera collected from patients with liver disease who also had high alkaline phosphatase activities. In contrast, the observed concentration increased steadily in serum with normal alkaline phosphatase activity. In the normal pool supplemented with $15.0 \mu \mathrm{g} / \mathrm{ml}$ FPHT (as PHT equivalent). When added alkaline phosphatase to the normal serum pool, we observed rapid conversion of FPHT into PHT within $10 \mathrm{~min}$, but the concentrations then declined with longer incubation time. However, when we repeated the experiment with protein-free ultrafiltrate, we observed rapid conversion of FPHT to PHT, but the concentration did not decline with longer incubation time. ${ }^{11}$ The i.v. FPHT is used when oral PHT loading is possible. The patients with seizures who could receive oral FPHT loading (awake on arrival, alert, no emesis, and lack of end tracheal intubation, repeated seizures, or SE after arrival). The outcomes included the number of patients receiving i.v. FPHT who could have received oral PHT loading. The FPHT administration is inappropriate almost half the time. Oral PHT loading is less expensive and safe. ${ }^{12}$ The novel immunoreactive metabolite derived from FPHT that has been hypothesized previously as present in sera from renally impaired patients receiving this prodrug. The metabolite of PHT, Mass fragments associated with glucuronic acid were also present. This metabolite demonstrated immunoreactivity sufficient to lead to falsely increased reported values for PHT immunoassays. The observed immunoreactivity was also proportional to the relative concentration of the metabolite in collected fractions. The presence of phenyl groups with chemical shifts identical to those of PHT, as well as the presence of a methylene bridge, which was consistent with the same methylene bridge present on the phosphate ester of FPHT. Comparative analysis of serum samples from renally impaired patients receiving PHT vs FPHT using multiple reaction monitoring quantification demonstrated that this metabolite was associated with FPHT administration. A unique immunoreactive oxymethylglucuronide metabolite derived from FPHT has been isolated from sera from uremic patients receiving this prodrug. ${ }^{13}$ The FPHT, is useful for acute seizures, is given by parenteral administration, and has few cardiac and local irritation adverse effects. There is limited experience in the administration of this new agent to newborns, and concern has been raised regarding the conversion of the prodrug to PHT. In two low-birth-weight infants, it was observed that FPHT was converted adequately with varying effects on seizure control. ${ }^{14}$ Fosphenytoin (FPHT/Cerebyx) is well absorbed when given i.m. Pharmacokinetic studies had the first plasma sample obtained $30 \mathrm{~min}$ after i.m. administration. The rate and extent of FPHT absorption and to evaluate the tolerability of i.m. FPHT compared with IM saline. Total PHT serum concentrations $10 \mu \mathrm{g} / \mathrm{mL}$ were obtained in $5 \mathrm{~min}$ in $14.3 \%$ of patients and in $26.3 \%$ after $10 \mathrm{~min}$. More than half the patients had therapeutic serum concentrations at $30 \mathrm{~min} ; 45.8 \%$ of patients reported no pain at either the FPHT or saline injection site. No significant difference in pain was noted between FPHT and saline injection sites at 60 min and thereafter. Early decrease in blood pressure occurred. Classic PHTinduced CNS side effects were evident in one third of patients within $1 \mathrm{~h}$ after injection. i.m. FPHT is rapidly absorbed, i.m. FPHT is well tolerated by most patients irrespective of injection volume. ${ }^{15}$ The value of FPHT compared with PHT for treating patients following a seizure. PHT to be the preferred option compared with FPHT. The PHT was very robust. ${ }^{16}$ 
Tonic-clonic status epilepticus (TCSE) is the most common neurological emergency. The risk of complications increases substantially as TCSE lasts longer than 60 minutes. Ideally, drugs used to treat this condition should be well tolerated when administered as rapid i.v. infusions and should not interfere with patients' state of consciousness or cardiovascular and respiratory functions. Because of its efficacy, absence of sedation or respiratory suppression, I.V PHT has largely replaced phenobarbital as the second agent of choice (benzodiazepine) in the treatment of TCSE. While the efficacy of PHT in the treatment of acute seizures and TCSE is well established, the parenteral formulation of PHT has several inherent shortcomings which compromise its tolerability and limit the rate of administration. i.v. PHT has been associated with fatal haemodynamic complications and serious reactions at the injection site including skin necrosis and amputation of extremities. FPHT, has the same pharmacological properties as PHT but none of the injection site and cardiac rhythm complications of i.v. infusions of PHT. While FPHT costs more than i.v. PHT, treating the acute and chronic complications of TCSE itself and the complications of i.v. PHT can also be costly. All other factors being equal, there is no doubt that FPHT is better tolerated and can be delivered faster than i.v. PHT; 2 measures that clearly improve outcome in patients with TCSE. The tolerability of i.m. FPHT also extends its use to clinical situations where prompt administration of a nondepressing AE is indicated but secure I.V access. ${ }^{17}$ The pharmacoeconomic of PHT and FPHT alone and in combination in hospitalized patients, the PHT and FPHT administration, frequency of adverse reactions, methods of treating adverse reactions, and demographic information. If $50 \%$ of PHT loading doses were substituted with FPHT, a reduction in adverse events. If PHT maintenance dosages were substituted with FPHT. It appears that FPHT reduces adverse events at a reasonable increase in total hospital costs. ${ }^{18}$ An acute toxic interaction has been described, in which sublethal doses of pyridostigmine bromide (PB) and the insect repellent N,N-diethyl-m-toluamide (DEET), when administered concomitantly, resulted in seizures and lethality. The possible relationships between seizures and lethality and the role of the cholinergic system in this interaction, PB ( $5 \mathrm{mg} / \mathrm{kg})$, DEET (200 $\mathrm{mg} / \mathrm{kg})$ or PB $(3 \mathrm{mg} / \mathrm{kg})+$ DEET $(200 \mathrm{mg} / \mathrm{kg})$ were administered i.p. to male ICR mice, alone or following i.p. pretreatment, with one of several AEDs: diazepam, $10 \mathrm{mg} / \mathrm{kg}$; FPHT, $40 \mathrm{mg} / \mathrm{kg}$; phenobarbital, $45 \mathrm{mg} / \mathrm{kg}$; or dextrophan, $25 \mathrm{mg} / \mathrm{kg}$ ), or the anticholinergic agents, atropine $(5 \mathrm{mg} / \mathrm{kg})$, atropine methyl nitrate $(2.7 \mathrm{mg} / \mathrm{kg})$, or mecamylamine $(2.5 \mathrm{mg} / \mathrm{kg})$. The AEs selected for this study act through different mechanisms to reduce seizures. None of the AEs was able to reduce the incidence of seizures following treatment with $\mathrm{PB}$, DEET or PB+DEET. Only diazepam delayed the onset of seizures. FPHT or diazepam significantly prolonged the time to lethality following PB, but only FPHT reduced the incidence of PB-induced lethality. Diazepam or phenobarbital significantly prolonged the time to lethality following $\mathrm{PB}+\mathrm{DEET}$. Both atropine and atropine methyl nitrate protected against $\mathrm{PB}$ and $\mathrm{PB}+\mathrm{DEET}$-induced lethality and PB-induced seizures. Neither agent blocked seizures resulting from DEET or PB+DEET. Mecamylamine reduced seizures and lethality in PB-treated mice, but not in mice treated with DEET or PB+DEET. The results indicate that seizure activity is not a causative factor in the toxic interaction between PB and DEET. Furthermore, PB, DEET and $\mathrm{PB}+\mathrm{DEET}$ induce seizures that are resistant to standard AEs, and each appears to operate through different mechanisms to produce seizures. Peripheral muscarinic receptors may play a specific role in lethality caused by PB+DEET. ${ }^{19}$ FPHT is a water-soluble disodium phosphate ester of PHT, with AE properties. The neuroprotective properties in a cardiac arrest-induced global ischemia model, After 12 minute ischemia, Long-Evans hooded rats were resuscitated, given FPHT ( $30 \mathrm{mg} / \mathrm{kg}$, i.m.) or saline 5 minutes after the ischemic episode, and killed on day 7. Brains were removed, fixed, and vibrato me sectioned to assess the numbers of normal appearing CAI pyramidal neurons and for immunohistological staining of glial fibrillary acidic protein (GFAP). After global ischemia, the number of hippocampal CA1 pyramidal neurons decreased significantly. Most hippocampal CA1 pyramidal neurons showed signs of injury and GFAP immunoreactivity of the region increased. With FPHT treatment 5 min after ischemia, hippocampal CA1 pyramidal neurons remained at near control level, however, GFAP staining was not significantly changed. Although indicating different neuronal and glial responses following FPHT treatment, nevertheless, suggest that FPHT is an effective neuroprotectant against ischemia-induced damage. ${ }^{20}$

The pharmacokinetic behavior of FPHT, has been characterized in normal subjects. The effect of hepatic or renal disease on the rate and extent of conversion of FPHT to PHT. The mean time to reach the peak plasma FPHT concentration was similar for each of the three groups. However, the mean time to achieve peak plasma concentrations of PHT tended to occur earlier in the hepatic or renal disease groups than in healthy subjects. The half-life of FPHT was $4.5,9.2$, and $9.5 \mathrm{~min}$ for the three groups, respectively. There was a trend toward increased FPHT clearance and earlier peak PHT concentration in subjects with hepatic or renal disease. This finding is consistent with decreased binding of FPHT to plasma proteins and increased fraction of unbound FPHT resulting from decreased plasma protein concentrations associated with these disease states. The conversion of FPHT to PHT was equally efficient in subjects with hepatic or renal disease and healthy subjects. The differences in pharmacokinetic parameters between the three groups were not statistically significant, FPHT administration to patients with hepatic or renal disease. To minimize the incidence of adverse effects in this patient population, FPHT may need to be administered at lower doses or infused more slowly. ${ }^{21}$ The administration of PHT suspension in conjunction with enteral nutrition supplements through nasogastric (NG) feeding tubes to humans has been associated with suboptimal PHT absorption, subtherapeutic concentrations, and breakthrough seizures. Postulated mechanisms include chelation to proteins and electrolytes in the enteral feeding, binding to NG tubing, and alterations in gastrointestinal $\mathrm{pH}$ resulting in precipitation of PHT. The purpose of this pilot study was to evaluate the oral absorption of commercially available FPHT injectable solution compared to PHT suspension in the rat to determine whether equivalent oral FPHT and PHT suspension doses should be used for future human studies of FPHT oral absorption in the presence of concomitant enteral nutrition. A single oral $30 \mathrm{mg} / \mathrm{kg}$ PHT equivalents dose of either commercially available FPHT or PHT suspension was administered to male Wistar rats. No significant differences in AUC last were found. The presence of a phosphate ester moiety does not appear to inhibit the appearance of PHT following oral administration of FPHT. PHT plasma concentration profiles following oral administration of FPHT are characterized by higher $\mathrm{Cmax}$ and shorter Tmax values relative to oral administration of PHT suspension. ${ }^{22}$ The pharmacologic activity of FPHT, is due to in vivo conversion to PHT. FPHT concentrations cannot be accurately estimated by PHT immunoassays owing to the nonlinear relation between FPHT concentration and the observed cross-reactivity. The problem of slow conversion of FPHT to PHT in serum in vitro can be circumvented by rapidly converting FPHT 
to PHT in vitro by alkaline phosphatase. Drug-free serum, heparin, EDTA, or citrated plasma was supplemented with 2 concentrations of FPHT. The specimens were incubated, and PHT concentrations were measured by fluorescence polarization and chemiluminescent assays. In the absence of enzyme, we observed little conversion of FPHT to PHT, but in the presence of only $10 \mu \mathrm{L}$ of enzyme, the conversion of FPHT to PHT was complete in 5 minutes. We also observed complete conversion of FPHT to PHT by alkaline phosphatase in heparin, EDTA, and citrated plasma. If clinically indicated, the PHT concentration can be measured before and after addition of enzyme to roughly estimate the rate of conversion. ${ }^{23}$ Measure the brain penetration of PHT after I.V administration of either standard PHT or FPHT. The time required for FPHT to be converted to PHT in the bloodstream would delay the accumulation of PHT in brain. Although the initial PHT-free fraction was significantly higher for FPHT-treated rats than it was for PHT-treated rats, brain PHT levels were significantly reduced after infusion of FPHT. When FPHT is used for treatment of generalized SE, it should be anticipated that lower initial brain PHT levels will be achieved than are typically found with standard PHT. ${ }^{24}$

In comparison with PHT preparations, which have a $\mathrm{pH}$ value of 11, FPHT, a phosphorylated prodrug of PHT, has a $\mathrm{pH}$ value of only 8.6 , which decreases the risk of cardiovascular and cutaneous side effects. The near-neutral $\mathrm{pH}$ value of FPHT allows effective I.V or I.M administration. A 1-mg PHT equivalent (PE) of FPHT is converted to $1 \mathrm{mg}$ of PHT in adults. We describe four infants whose seizures were treated with I.V FPHT. We had difficulty maintaining therapeutic serum PHT levels of 10 to $20 \mu \mathrm{g} / \mathrm{mL}$ on doses of 5 to $8 \mathrm{mgPE} / \mathrm{kg} / \mathrm{day}$, and many bolus doses of 5 to $10 \mathrm{mgPE} / \mathrm{kg}$ or maintenance doses of more than $10 \mathrm{mgPE} / \mathrm{kg} / \mathrm{day}$ were given. Despite increased doses in three out of the four patients, a therapeutic serum PHT level was not maintained. ${ }^{25}$ Impaired protein binding of PHT in uremia which was prompted us to investigate the protein binding of FPHT in uremic sera. The role of uremic toxins hippuric acid and indoxyl sulfate as potential inhibitor of the protein binding of FPHT because these compounds impair protein binding of PHT in uremia. Five serum pools were prepared from normal volunteers and five pools from patients with uremia. None of them received PHT. The normal serum pools were diluted with saline to mimic the albumin concentration of uremic pool. Both the diluted normal pool and the uremic pool were supplemented with FPHT; after incubation at room temperature for 30 minutes, total and free FPHT concentrations as PHT equivalents were measured. The significantly elevated free FPHT concentration in uremic sera compared with that of normal sera in all cases. Because both normal and uremic sera had the same concentrations of albumin, the elevated free FPHT concentration in uremic sera was not caused by hypoalbuminemia. Both indoxyl sulfate and hippuric acid were caused significant displacement of PHT from protein binding. In contrast, none caused any displacement of FPHT from protein binding. ${ }^{26}$ Fosphenytoin (FPHT or Cerebyx), is a water soluble prodrug that is rapidly and completely converted to PHT. The injection-site tolerance and safety of I.M FPHT (>10 mg/kg doses) requiring a PHT loading dose. Patients received injections at single or multiple sites with volumes ranging from 4 to $30 \mathrm{ml}$ per injection site. The majority of patients had no irritation (erythema, swelling, tenderness, bruising) or complaints of discomfort related to FPHT injection either after injection (95\%) or at follow-up (88\%). Irritation, when reported, was mild in all cases. Forty of 60 patients $(67 \%)$ reported transient side effects, primarily involving the central nervous system, such as nystagmus, dizziness or ataxia, which are commonly associated with PHT therapy. All patients received prescribed doses; no patient had an injection(s) stopped due to intolerance or side effects. No serious adverse events occurred with I.M FPHT. The I.M FPHT was demonstrated to be a safe and well tolerated, and in many instances, a preferable alternative to other means of PHT loading. ${ }^{27}$ The cross-reactivity of FPHT, cross-reactivity utilized in vitro correlation of the two immunoassays with a validated. In vitro data were used to predict "immunoassay-derived" PHT concentrations in plasma samples collected from actual patients after I.V or I.M FPHT dosing. The greatest degree of PHT concentration overestimation occurred at times when FPHT concentrations were highest: within 1 to $2 \mathrm{~h}$ after i.v. infusion or during the first 2 to $4 \mathrm{~h}$ after i.m. injection. It is recommended that PHT concentrations not be monitored using these or other potentially nonspecific immunoanalytical methods for at least $2 \mathrm{~h}$ after i.v. FPHT infusion or $4 \mathrm{~h}$ after i.m. FPHT injection. ${ }^{28}$

The PHT has been reported to exert variable AE effects in the complex partial seizures. PHT is only water soluble at a $\mathrm{pH}$ of more than 10, and it has been suspected that poor absorption of the drug. Recently, FPHT, has been developed by phosphorylating PHT which makes the drug water soluble at physiological $\mathrm{pH}$ while it is rapidly transformed to PHT after injection. The AE profile and the absorption after I.P injection of FPHT, compared to its parental drug PHT. The pharmacokinetic parameters of PHT and FPHT were compared by determining plasma levels of PHT after i.p. injection of $50 \mathrm{mg} / \mathrm{kg}$ PHT or the equivalent dose of $84 \mathrm{mg} / \mathrm{kg}$ of FPHT in non-kindled female Wistar rats. After both injections the maximal plasma concentration of PHT was about $30 \mu \mathrm{g} / \mathrm{ml}$. The relative bioavailability of FPHT was $83 \%$. In contrast to PHT, failed injections resulting in non-detectable plasma concentration of PHT were almost absent after FPHT. The FPHT dose-dependently increased the focal seizure threshold. Seizure severity and duration at threshold were reduced only after the highest does of FPHT tested $(84 \mathrm{mg} / \mathrm{kg})$. Thus, FPHT showed AE properties similar to PHT. We conclude that FPHT is an adequate and reliable substitute for the parenteral injection of PHT in experimental seizure models of rats. ${ }^{29}$ In general, the new drug, FPHT offers an attractive alternative for parenteral PHT in select individuals. ${ }^{30}$ FPHT, is rapidly and completely converted to PHT in adults after I.V or I.M administration and is significantly better tolerated than parenteral PHT. FPHT is highly plasma-protein bound and, when present in sufficient concentration, will displace PHT from plasma proteins. The clinical utility is that FPHT may be used to achieve therapeutic PHT concentrations more rapidly than I.V. PHT infused at its maximum recommended rate. In a studies, FPHT maintained seizure control when substituted for oral PHT and for seizure prophylaxis in neurosurgery and trauma patients. Adverse events associated with FPHT generally were related to the CNS and were similar to those associated with PHT, except for a higher incidence of transient pruritus with FPHT. Intravenous (I.V) FPHT has significant advantages over I.V PHT: It requires a shorter infusion time and fewer I.V disruptions, causes less pain and burning at the infusion site and minimal consequences in case of I.V infiltration, allows longer maintenance of I.V sites, and has better I.V fluid compatibility and stability. In contrast to I.M PHT, I.M FPHT is well tolerated in both large loading doses and maintenance doses. ${ }^{31}$ FPHT was developed as a replacement for sodium PHT. After absorption, PHT is cleaved from FPHT. Unlike PHT, FPHT is freely soluble in aqueous solutions and rapidly absorbed by the I.M route. FPHT has been tested successfully for three indications in humans: I.M maintenance dosing, I.M loading dose administration and I.V treatment of SE. Local toxicity (pain, burning, itching) is less by 
the I.M or I.V route for FPHT than for standard injectable sodium PHT. Systemic toxicity is similar with both preparations except that hypotension is less common and paresthesias are more common with FPHT. $^{32}$

The protein binding of FPHT, prodrug of PHT sodium, to human serum proteins, serum albumin and alpha 1-acid glycoprotein was determined. FPHT was weakly bound to alpha 1-acid glycoprotein $(13.3 \%)$. Simultaneous incubation with high concentrations of carbamazepine $(10 \mu \mathrm{g} / \mathrm{ml})$ and diazepam $(5 \mu \mathrm{g} / \mathrm{ml})$ or therapeutic concentrations of PHT $(10 \mu \mathrm{g} / \mathrm{ml})$ had no effect on the binding of FPHT to human serum proteins. High concentrations of phenobarbital $(160 \mu \mathrm{g} / \mathrm{ml})$, PHT $(50 \mu \mathrm{g} / \mathrm{ml})$, or valproic acid $(500 \mu \mathrm{g} / \mathrm{ml})$, however, caused slight, but significant, increases in the free fraction of FPHT in serum protein. Phenylbutazone and sulfisoxazole resulted in a $48 \%$ increase in FPHT free fraction while warfarin had a slight (8\%), but significant, increase in free fraction of FPHT. It was concluded that the concentration of albumin was the most important determinant for the plasma free fraction of FPHT in man. Potential increase in FPHT clearance may be observed in hypoalbuminemia. ${ }^{33}$ The compare the frequency, severity, and time course of venous irritation after administration of a single I.V dose of PHT with an equimolar dose of FPHT. Volunteers randomly received a 30-minute infusion of PHT sodium $250 \mathrm{mg}(250 \mathrm{mg} / 5 \mathrm{ml})$ or an equimolar dose of FPHT 375 $\mathrm{mg}(375 \mathrm{mg} / 5 \mathrm{ml})$. Subjects assessed venous irritation (pain, burning, itching), and investigators evaluated phlebitis (erythema, swelling, tenderness), induration, exudation, and cording. PHT was associated with a significantly higher degree of pain at the infusion site in all subjects and a significant degree of phlebitis in eight subjects; cording occurred in six subjects. The time course of PHT-induced phlebitis was bimodal. Erythema and tenderness were prominent at the end of the infusion and again at 24 hours. Cording was first noted between 24 hours and 1 week after infusion. In contrast, FPHT was associated with mild pain in two subjects, one incident of phlebitis, and no erythema or cording. FPHT administration resulted in significantly less venous irritation and phlebitis compared with an equimolar dose of PHT. The clinical use of this water-soluble PHT prodrug should minimize the frequency and severity of infusion-site reactions and should allow convenient, rapid, i.v. administration of drug, undiluted or admixed with i.v. solutions. ${ }^{34}$

The FPHT sodium and its safety is compared with that of PHT. After i.v. or i.m. injection, FPHT, is rapidly hydrolyzed to PHT. FreePHT concentrations equivalent to those obtained with i.v. PHT can be achieved with FPHT given at equimolar loading doses by selecting the appropriate rate of FPHT administration. FPHT can be expected to interact with the same drugs that interact with PHT. The dosage is expressed as PHT sodium equivalents (PE). The standard loading dose for adults with SE is $15-20 \mathrm{mg} \mathrm{PE} / \mathrm{kg}$ i.v. infused at $100-150$ $\mathrm{mg} / \mathrm{min}$; i.m. administration is not recommended for this condition. For nonemergency situations, a $10-$ to $20-\mathrm{mg} \mathrm{PE} / \mathrm{kg}$ loading dose can be given i.v. or i.m. FPHT has advantages over PHT injection that are related to its greater aqueous solubility, which obviates the extreme alkalinity, propylene glycol, and ethanol needed in the injectable PHT formulation. Intravenous (I.V) FPHT has been associated with less soft-tissue injury and fewer adverse effects in general than PHT. FPHT, when administered i.m., is completely absorbed, is relatively well tolerated, and provides more predictable serum drug concentrations than i.m. PHT. FPHT offers practical and clinical advantages over i.v. PHT. ${ }^{35}$ FPHT is a PHT prodrug, was designed to overcome many of the shortcomings associated with parenteral PHT sodium. Specifically, FPHT is a highly water-soluble, that has no known pharmacologic activity before its conversion to PHT. Dosing of FPHT uses PHT equivalents (PE) to minimize dosage errors when converting from the conventional formulation. Pharmacokinetic studies documented that the agent is rapidly and completely converted to PHT after i.v and i.m dosing. Reported conversion half-lives after i.v administration range from $8-15$ minutes. The absorption rate appears to be the rate-limiting step in the conversion of FPHT to PHT after i.m administration (half-life range $22-41 \mathrm{~min}$ ). Bioavailability of PHT derived from both i.v and i.m FPHT is essentially $100 \%$. As a consequence of concentration-dependent protein binding, FPHT is bioequivalent to PHT sodium at I.V infusion rates of $100-150 \mathrm{mg}$ $\mathrm{PE} / \mathrm{minute}$ and $50 \mathrm{mg} /$ minute, respectively. In clinical studies to date, FPHT is safe and significantly better tolerated than PHT sodium when administered i.v. It is also well tolerated when given i.m, and this is a valuable alternative route of administration when i.v access or cardiographic monitoring is unavailable. Its pharmacoeconomic advantages over PHT have not been documented in formal studies to date, although the likelihood of savings based on cost-effectiveness analyses is high. Hence, FPHT has the potential as a safe, welltolerated, and effective means of delivering PHT parenterally in a variety of clinical settings. ${ }^{36}$ Safety, tolerability, and pharmacokinetics of FPHT sodium, were investigated after a temporary substitution of I.M FPHT for oral PHT sodium in epileptic or neurosurgical patients taking oral PHT sodium (100-500 mg/d). Both groups had similar types and frequencies of mild to moderate adverse events. FPHT was as well tolerated as i.m. placebo at the injection site. i.m. FPHT equimolar to a patient's oral PHT dose produced equal or greater plasma PHT concentrations. Dosing adjustments are not required when i.m FPHT is temporarily substituted or oral PHT therapy is resumed. i.m. M FPHT is a safe and well-tolerated alternative to oral PHT when oral administration is not feasible. ${ }^{37}$

The safety, tolerability, and pharmacokinetic profile of FPHT after i.m and i.v administration. The i.m. FPHT was safe and well tolerated, with no irritation found for $99 \%$ of all injection site evaluations. Adverse events associated with the drug occurred in $9 \%$ of patients, commonly those typical of the parent drug. For i.v. treatment, the frequency of mild irritation at the infusion site was significantly lower in the FPHT group (6\%) than in the PHT group (25\%). Reductions in infusion rates were required in $17 \%$ and $36 \%$ of FPHT and PHT recipients, respectively. No significant difference was observed relative to adverse events or seizure frequency between the groups. Trough plasma PHT concentrations were approximately $10 \mu \mathrm{grams} /$ $\mathrm{ml}$ or greater in patients receiving at least 3 days of i.m. and i.v. FPHT. Trough PHT concentrations were similar between patients receiving I.V PHT and FPHT on all study days. FPHT can be administered i.v. and i.m. in neurosurgical patients to achieve and maintain therapeutic PHT concentrations for up to 14 days. Both routes are safe and well tolerated. Intravenous (I.V) FPHT is significantly better tolerated than I.V PHT sodium in this patient subset. ${ }^{38}$ FPHT sodium, a prodrug of PHT, was developed as a replacement for parenteral PHT sodium. Unlike PHT, FPHT is freely soluble in aqueous solutions, including standard I.V. solutions, and is rapidly absorbed by the i.m. route. FPHT is metabolized (half-life of 8 to $15 \mathrm{~min}$ ) to PHT by endogenous phosphatases. Therapeutic unbound and total plasma PHT concentrations are consistently attained after i.m. or i.v. administration of FPHT loading doses. FPHT has fewer local adverse effects (e.g., pain, burning, and itching at the injection site) after i.m. or i.v. administration than parenteral PHT. Systemic effects related to 
the CNS are similar for both preparations, but transient paresthesias are more common with FPHT. ${ }^{39}$

Phenobarbital, diazepam, lorazepam, and PHT are all currently used for the treatment of acute seizures, including SE. None of these drugs is considered ideal. FPHT is a new PHT prodrug that fulfills many of the properties of an ideal AED. The safety, tolerance, and pharmacokinetics of i.m. administered FPHT have been evaluated in three clinical trials involving patients requiring loading or maintenance doses of PHT. These investigations demonstrated that FPHT is rapidly and completely absorbed after injection into muscle and is quickly converted to produce therapeutic PHT plasma concentrations. Plasma concentrations of PHT achieved with i.m. FPHT exceeded those associated with an equimolar dose of oral PHT. i.m. FPHT was well tolerated both locally and systemically. Only mild and transient reactions occurred at the injection site. The most common systemic adverse events reported-somnolence, nystagmus, dizziness, and ataxia-are side effects commonly seen with PHT and tended to be mild. Preexisting seizure disorders remained stable. Combination treatment with i.v. diazepam or lorazepam to attain rapid seizure control and i.m. FPHT to maintain the AE effect theoretically offers many advantages for control of acute seizures and should be studied ${ }^{40}$ FPHT is a water-soluble disodium phosphate ester of PHT that is converted in plasma to PHT. FPHT is compatible with most common i.v. solutions and can be administered safely through the i.m. route. An additional safety factor is the absence of propylene glycol in the FPHT formulation. Propylene glycol is used as a vehicle in the i.v. PHT preparation and by itself may produce serious cardiovascular complications. Studies of the pharmacokinetics, safety, and tolerance of i.v. FPHT have demonstrated that FPHT produces PHT plasma concentrations similar to those achieved with oral and i.v. PHT, but without significant cardiovascular effects and only minimal discomfort at the injection site. Aside from local reactions, the most common adverse events associated with FPHT have been pruritus and reactions typical of PHT (e.g., dizziness, somnolence, and ataxia). FPHT represents a significant advance in the treatment of patients with seizures who require parenteral therapy. ${ }^{41} \mathrm{PHT}$ is widely used for the prevention and treatment of acute seizures in children. Although it has the advantage of being available in parenteral form, it cannot be given through the i.m. route. Furthermore, problems with venous accessibility and maintenance may complicate i.v. administration of PHT in newborns and very sick infants. FPHT can be safely administered through the i.m. route, and, because of the physical characteristics of its formulation, it offers advantages over PHT for i.v. administration. Clinical studies with i.v. and i.m. FPHT demonstrate that the efficacy, safety, and pharmacokinetics of this drug are similar in 5- to 18-year-old children and in young adults. The safety and pharmacokinetic profile of i.v. and i.m. FPHT in younger children and infants is currently being investigated. ${ }^{42}$ FFPHT is a prodrug that is highly soluble in i.v. solutions without olubilizing agents and is supplied in vials for i.v. use. FPHT is converted to PHT following parenteral administration. It is very effective in terminating seizures and will stop most SE episodes and provide long-term control without any decreased level of consciousness. All of these drugs should be administered slowly to avoid respiratory depression and apnea.

Several antiepileptic drugs (AEDs) have been tested, recently, AEDs are include vigabatrin, oxcarbazepine, lamotrigine, felbamate and gabapentin. In addition to these, additional drugs are flunarizine,
FPHT and stiripentol. Midazolam recently has shown effectiveness in the treatment of SE. Flunarizine is a selective calcium channel blocker that has shown AE properties. It is a long-acting AE that clinical studies have shown to have effects similar to those of PHT and carbamazepine in the treatment of partial, complex partial and generalised seizures. FPHT was developed to eliminate the poor aqueous solubility and irritant properties of I.V PHT. It is rapidly converted to PHT after I.V or I.M administration. This prodrug showed minimal evidence of adverse events and no serious cardiovascular or respiratory adverse reactions. It may have a clear advantage over the present parenteral formulation of PHT. Midazolam is a benzodiazepine that is more potent than diazepam as a sedative, muscle relaxant and in its influence on electroencephalographic measures. It has been shown to be an effective treatment for refractory seizures in SE. Stiripentol has AE properties as well as the ability to inhibit the cytochrome P450 system. There are significant metabolic drug interactions between stiripentol and PHT, carbamazepine and phenobarbital (phenobarbitone). Stiripentol has been studied in patients with partial seizures, refractory epilepsy and refractory absence seizures with some efficacious results. ${ }^{43}$

\section{Pharmacokinetic properties}

PHT is available in two types of oral formulations that differ in their pharmacokinetics: rapid-release and extended-release forms. Once-daily dosing is possible only with the extendedrelease formulations, and due to differences in dissolution and other formulation-dependent factors, the plasma PHT level may change when converting from one formulation to another. Confusion also can arise because different formulations can include either PHT or PHT sodium. Therefore, comparable doses can be approximated by considering "PHT equivalents," but serum level monitoring is also necessary to assure therapeutic safety. The pharmacokinetics of PHT are influenced markedly by its binding to serum proteins, by the nonlinearity of its elimination kinetics, and by its metabolism by CYPs. PHT is extensively bound (about $90 \%$ ) to serum proteins, mainly albumin. Small variations in the percentage of PHT that is bound dramatically affect the absolute amount of free (active) drug; increased proportions of free drug are evident in the neonate, in patients with hypoalbuminemia, and in uremic patients. Some agents, such as valproate, can compete with PHT for binding sites on plasma proteins; when combined with valproate-mediated inhibition of PHT metabolism, marked increases in free PHT can result. Measurement of free rather than total PHT permits direct assessment of this potential problem in patient management. PHT is one of the few drugs for which the rate of elimination varies as a function of its concentration (i.e., the rate is nonlinear). The plasma half-life of PHT ranges between 6 and 24 hours at plasma concentrations below $10 \mu \mathrm{g} / \mathrm{ml}$ but increases with higher concentrations; as a result, plasma drug concentration increases disproportionately as dosage is increased, even with small adjustments for levels near the therapeutic range. The majority (95\%) of PHT is metabolized principally in the hepatic endoplasmic reticulum by CYP2C9/10 and to a lesser extent CYP2C19. The principal metabolite, a parahydroxyphenyl derivative, is inactive. Because its metabolism is saturable, other drugs that are metabolized by these enzymes can inhibit the metabolism of PHT and increase its plasma concentration. Conversely, the degradation rate of other drugs that are substrates for these enzymes can be inhibited by PHT; one such drug is warfarin, and addition of PHT to a patient receiving 
warfarin can lead to bleeding disorders. An alternative mechanism of drug interactions arises from PHT's ability to induce diverse CYPs; coadministration of phenytoin and medications metabolized by these enzymes can lead to an increased degradation of such medications. Of particular note in this regard are oral contraceptives, which are metabolized by CYP3A4; treatment with PHT could enhance the metabolism of oral contraceptives and lead to unplanned pregnancy. The potential teratogenic effects of PHT underscore the importance of attention to this interaction. Carbamazepine, oxcarbazepine, phenobarbital, and primidone also can induce CYP3A4 and likewise might increase degradation of oral contraceptives. The low aqueous solubility of phenytoin hindered its I.V use and led to production of FPHT. The FPHT is converted into PHT by phosphatases in liver and red blood cells with a half-life of 8 to 15 minutes. FPHT is extensively bound ( $95 \%$ to $99 \%$ ) to human plasma proteins, primarily albumin. This binding is saturable and FPHT displaces PHT from protein binding sites. FPHT is useful for adults with partial or generalized seizures when I.V or I.M administration is indicated. ${ }^{44-48}$

The water-soluble PHT derivatives, bearing an ionizable group, results showed that hydrolysis of amino esters is very fast, much more than that of FPHT. ${ }^{49}$ FPHT is a phosphate ester prodrug of PHT developed as a replacement for standard injectable sodium PHT. After absorption, PHT is cleaved (conversion half-life $8-15 \mathrm{~min}$ ) from FPHT. Unlike PHT, FPHT is freely soluble in aqueous solutions and rapidly absorbed by the i.m route. FPHT has been tested successfully for three indications in humans: i.m maintenance dosing, i.m loading dose administration and i.v treatment of SE. Local toxicity (pain, burning, itching) is less by the i.m or i.v route for FPHT than for injectable sodium PHT. FPHT is a highly water-soluble, phosphate ester of PHT that has no known pharmacologic activity before its conversion to PHT. It is rapidly and completely converted to PHT after i.v and i.m dosing, half-lives after i.v administration range from $8-15$ minutes. The absorption rate appears to be the rate-limiting step in the conversion of FPHT to PHT after i.m administration (half-life range $22-41 \mathrm{~min}$ ). Bioavailability of PHT derived from both i.v and i.m FPHT is essentially $100 \%$. As a consequence of concentrationdependent protein binding, FPHT is bioequivalent to PHT sodium at i.v infusion rates of $100-150 \mathrm{mg} \mathrm{PE} /$ minute and $50 \mathrm{mg} /$ minute, respectively. The FPHT is safe and significantly better tolerated than PHT sodium when administered i.v. It is also well tolerated when given i.m, and this is a valuable alternative route of administration when i.v access. Hence, FPHT has the potential as a safe, well-tolerated, and effective means of delivering PHT parenterally in a variety of clinical settings. The protein binding and pharmacokinetics of diazepam, FPHT (PHT prodrug), and PHT were evaluated. No significant differences were observed in the fraction unbound or pharmacokinetic parameters of FPHT, PHT, or diazepam when FPHT was administered alone compared to concomitant administration with diazepam. The PHT fraction unbound increased significantly with increased concentrations of FPHT, indicating displacement of PHT from its binding sites by FPHT. FPHT also demonstrated concentration dependent binding. The lack of a significant pharmacokinetic drug interaction between FPHT and diazepam suggests that these drugs may be safely administered together. ${ }^{50}$ FPHT is a water-soluble investigational PHT prodrug for parenteral administration. The healthy male volunteers received PHT sodium $(250 \mathrm{mg} / 5 \mathrm{ml})$ and FPHT $(375 \mathrm{mg} / 5 \mathrm{ml})$ i.v. in $30 \mathrm{~min}$ in a randomized. The fraction of unbound converted PHT at the end of the prodrug infusion, in the presence of $44 \mu \mathrm{g} / \mathrm{ml} \mathrm{FPHT}$, was significantly higher than at $180 \mathrm{~min}$, when the concentration of FPHT was $0.1 \mu \mathrm{g} /$ ml. FPHT was shown to be a bioequivalent PHT prodrug exhibiting less irritation at the injection site than the PHT. ${ }^{51}$ Either PHT or FPHT was injected after the second generalized tonic-clonic seizure. Rats treated with FPHT had significantly poorer treatment outcomes than rats treated with PHT, although no differences were found in the concentration of PHT in plasma or brain 65 min after injection..$^{52} \mathrm{~A}$ PHT prodrug, FPHT, has more favorable physicochemical properties than PHT for parenteral administration. The pharmacokinetic profile of FPHT i.v. and im administration in adult patients receiving chronic oral PHT monotherapy. Each patient received a single i.v. dose of undiluted FPHT equivalent to their twice daily PHT dose (100-200 $\mathrm{mg}$ ). An equivalent dose of i.m. FPHT was administered in the gluteus maximums muscle one week later. Serial blood samples were obtained after each dose. ${ }^{53}$ FPHT with advantageous physicochemical properties, FPHT is rapidly converted enzymatically to PHT in vivo. FPHT and PHT sodium have equivalent AE activity against seizures induced by MES in mice following i.p., oral, or i.v. administration. The $\mathrm{ED}_{50}$ doses were $16 \mathrm{mg} / \mathrm{kg}$ for i.v. FPHT and $8 \mathrm{mg} / \mathrm{kg}$ for i.v. PHT sodium. FPHT and PHT sodium have similar antiarrhythmic activity against ouabain-induced ventricular tachycardia in anesthetized dogs. Only PHT sodium displayed in vitro antiarrhythmic activity against strophanthidin-induced arrhythmias in guinea pig right atria. In anesthetized dogs, a high dose of FPHT $(31 \mathrm{mg} / \mathrm{kg})$ was infused and the responses were compared to an equimolar dose of PHT sodium $(21 \mathrm{mg} / \mathrm{kg})$. The FPHT and PHT sodium treatments produced similar marked reductions in diastolic blood pressure and contractile force. The maximum effects of each treatment occurred at the time of maximum PHT sodium levels. The local irritation of FPHT was markedly less than PHT sodium following i.m. administration. ${ }^{54}$ The bioavailability of PHT from FPHT versus i.v administered sodium PHT was determined for i.v and I.M administration of FPHT to healthy volunteers. The bioavailability of FPHT is complete following i.v or i.m administration in single-dose volunteer studies. The absolute bioavailability of PHT derived from FPHT in subjects with therapeutic plasma PHT concentrations is being studied in patients given simultaneous infusions of stable isotope-labeled tracer doses of FPHT and sodium PHT. ${ }^{55}$ The PHT solution has many disadvantages like poor aqueous solubility. An approach to solve the problem is to prepared a phosphate ester of PHT (FPHT). This water-soluble compound is metabolized rapidly into $\mathrm{PO}_{4}$ and PHT. Patients received an i.v. or i.m. dose of FPHT at a dose equivalent to the patients' morning dose of PHT. After a period of 6 days, during which patients were again maintained with oral PHT, they were given a dose of FPHT via whichever route they had not yet received. The Tmax of the prodrug averaged 5.7 and $36 \mathrm{~min}$ after i.v. and i.m. administrations, respectively. The elimination half-life of FPHT (conversion to PHT) after i.v. and i.m. administration was 8.4 and 32.7 min, respectively, and both were independent of the dose. ${ }^{56}$ The prodrug FPHT and PHT in plasma samples, the concentration of PHT reached $90 \%$ of its maximum about 12 minutes after the end of the infusion of FPHT. At the dose of $1200 \mathrm{mg}$ of prodrug, the average peak concentration of PHT was about $17 \mu \mathrm{g} / \mathrm{mL}$, near the upper limit of the therapeutic range. Adverse reactions, lightheadedness, nystagmus, incoordination were minor and attributed to PHT. ${ }^{57}$

\section{Advantages and disadvantages of FPHT}

Advantages: More rapid i.v administration than PHT and no need for an in-line filter. It may be administered by i.m. injection. Lower potential for local tissue and cardiac toxicity than PHT. 
Associated with less pain and phlebitis at the injection site, fewer reductions in infusion rate and fewer changes of administration site because of injection site complications than PHT. Benefits in terms of ease of administration and improved tolerability vs PHT have pharmacoeconomic implications which may translate into an overall cost advantage.

Disadvantages: Approximately 10-fold higher acquisition cost vs PHT. FPHT is a parenterally administered prodrug of PHT, used in the treatment of patients with seizures. Advantages of FPHT over PHT include more rapid I.V administration, no need for an I.V filter, and a lower potential for local tissue and cardiac toxicity. Unlike PHT, FPHT may also be administered by i.m injection. Pharmacoeconomic data from a small study of patients with acute seizures showed an overall cost advantage of FPHT over PHT, despite a considerably greater acquisition cost of FPHT. The main cost drivers for PHT therapy were treatment costs associated with adverse events. In view of the limited pharmacoeconomic data currently available, it is in the interests of individual institutions to conduct their own formal pharmacoeconomic studies applying local cost data and patterns of clinical practise to determine whether FPHT should replace PHT on their formularly list. ${ }^{58}$

\section{Toxicity}

The toxic effects of PHT depend on the route of administration, the duration of exposure, and dosage. When FPHT, the water-soluble prodrug, is administered i.v. at an excessive rate in the emergency treatment of SE, the most notable toxic signs are cardiac arrhythmias, with or without hypotension, and/or CNS depression. Although cardiac toxicity occurs more frequently in older patients and in those with known cardiac disease, it also can develop in young, healthy patients. These complications can be minimized by administering FPHT at a rate of less than $150 \mathrm{mg}$ of PHT sodium equivalents per minute, a rate that therefore should not be exceeded. Acute oral over dosage results primarily in signs referable to the cerebellum and vestibular system; high doses have been associated with marked cerebellar atrophy. Toxic effects associated with chronic treatment also are primarily dose-related cerebellar-vestibular effects but also include other CNS effects, behavioral changes, increased frequency of seizures, gastrointestinal symptoms, gingival hyperplasia, osteomalacia, and megaloblastic anemia. Hirsutism is an annoying untoward effect in young females. Usually, these phenomena can be diminished by proper adjustment of dosage. Serious adverse effects, including those on the skin, bone marrow, and liver, probably are manifestations of drug allergy. Although rare, they necessitate withdrawal of the drug. Moderate elevation of the plasma concentrations of hepatic transaminases sometimes are observed; since these changes are transient and may result in part from induced synthesis of the enzymes, they do not necessitate withdrawal of the drug. Gingival hyperplasia occurs in about $20 \%$ of all patients during chronic therapy and is probably the most common manifestation of PHT toxicity in children and young adolescents. It may be more frequent in those individuals who also develop coarsened facial features. The overgrowth of tissue appears to involve altered collagen metabolism. Toothless portions of the gums are not affected. The condition does not necessarily require withdrawal of medication and can be minimized by good oral hygiene. A variety of endocrine effects have been reported. Inhibition of release of antidiuretic hormone (ADH) has been observed in patients with inappropriate ADH secretion. Hyperglycemia and glycosuria appear to be due to inhibition of insulin secretion. Osteomalacia, with hypocalcemia and elevated alkaline phosphatase activity, has been attributed to both altered metabolism of vitamin (vit) D and the attendant inhibition of intestinal absorption of $\mathrm{Ca}^{2+}$. PHT also increases the metabolism of vitamin $\mathrm{K}$ and reduces the concentration of vitamin $\mathrm{K}$-dependent proteins that are important for normal $\mathrm{Ca}^{2+}$ metabolism in bone. This may explain why the osteomalacia is not always ameliorated by the administration of vit-D. Hypersensitivity reactions include morbilliform rash in $2 \%$ to $5 \%$ of patients and occasionally more serious skin reactions, including Stevens-Johnson syndrome. Systemic lupus erythematosus and potentially fatal hepatic necrosis have been reported rarely. Hematological reactions include neutropenia and leukopenia. A few instances of red-cell aplasia, agranulocytosis, and mild thrombocytopenia also have been reported. Lymphadenopathy, resembling Hodgkin's disease and malignant lymphoma, is associated with reduced immunoglobulin A ( $\operatorname{IgA})$ production. Hypoprothrombinemia and hemorrhage have occurred in the newborns of mothers who received PHT during pregnancy; vit-K is effective treatment or prophylaxis. ${ }^{59-63}$

\section{Discussion}

The chemistry of nitrogen heteroatom containing organic compounds is becoming more popular in the area of research. Hydantoins and related compounds have shown good antiepileptic activities mainly against grand mal epilepsy. They bind to physiological targets or receptors, producing many possible mechanisms of actions. Hydantoins are inexpensive and easily synthesized and have been examined as AED. A slight variation in the substitution pattern on the hydantoin nucleus often causes a difference in activities. Therefore hydantoin with various substituents are being synthesized and tested for antiepileptic activities in search of better AEDs and great interest has arisen in the design and synthesis of new hydantoin compounds to explore their potent activities against epilepsy without or minimum side effects. ${ }^{64-66}$ The hydantoin nucleus has a useful structure for further molecular exploration for the development of new AEDs, has received much attention. In order to further explore chemical space available for pharmaceutical applications, there is a continued demand for the development of new hydantoins that have novel structures to AEDs development.

\section{Conclusion}

The hydantoin nucleus, due to its easy fictionalization at various position of ring has found considerable interest as the core structure of AEDs. The ring system became interesting in search of new and more potent AEDs with lesser side effect. The researchers have taken attention on hydantoin compounds due to its good antiepileptic potential. This therapeutical profile has attracted the attention of many researchers to explore this nucleus. By the present scenario it can be concluded that hydantoin have a great potential which remain to be disclosed till date. The antiepileptic profile of hydantoin represents much progress with regards.

\section{Acknowledgement}

None.

\section{Conflict of interest}

The authors declared that there is no conflict of interest. 


\section{Referencres}

1. Eriksson K, Keränen T, Kälviäinen R. Fosphenytoin. Expert Opin Drug Metab Toxicol. 2009;5(6):695-701.

2. Donough JH, Benjamin A, Monagle JD, et al. Effects of fosphenytoin on nerve agent-induced status epilepticus. Drug Chem Toxicol. 2004;27(1):27-39.

3. Gwer SA, Idro RI, Fegan G, et al. Fosphenytoin for seizure prevention in childhood coma in Africa: A randomized clinical trial. J Crit Care. 2013;28(6):1086-1092.

4. Rudis MI, Touchette DR, Swadron SP, et al. Cost-effectiveness of oral phenytoin, intravenous phenytoin, and intravenous fosphenytoin in the emergency department. Ann Emerg Med. 2004;43(3):386-397.

5. Wang X, Patsalos PN. A comparison of central brain (cerebrospinal and extracellular fluids) and peripheral blood kinetics of phenytoin after intravenous phenytoin and fosphenytoin. Seizure. 2003;12(6):330-336.

6. Ogutu BR, Newton CR, Muchohi SN, et al. Pharmacokinetics and clinical effects of phenytoin and fosphenytoin in children with severe malaria and status epilepticus. Br J Clin Pharmacol. 2003;56(1):112-119.

7. Applebaum J, Levine J, Belmaker RH. Intravenous fosphenytoin in acute mania. J Clin Psychiatry. 2003;64(4):408-409.

8. Fischer JH, Patel TV, Fischer PA. Fosphenytoin: clinical pharmacokinetics and comparative advantages in the acute treatment of seizures. Clin Pharmacokinet. 2003;42(1):33-58

9. Keegan MT, Bondy LR, Blackshear JL, et al. Hypocalcemia like electrocardiographic changes after administration of intravenous fosphenytoin. Mayo Clin Proc. 2002;77(6):584-586.

10. Muchohi SN, Kokwaro GO, Maitho TE, et al. Pharmacokinetics of phenytoin following intravenous and intramuscular administration of fosphenytoin and phenytoin sodium in the rabbit. Eur $J$ Drug Metab Pharmacokinet. 2002;27(2):83-89.

11. Dasgupta A, Schlette E. Rapid in vitro conversion of fosphenytoin into phenytoin in sera of patients with liver disease: role of alkaline phosphatase. J Clin Lab Anal. 2001;15(5):244-250.

12. Johnson J, Wrenn K. Inappropriate fosphenytoin use in the ED. Am J Emerg Med. 2001;19(4):293-294.

13. Annesley TM, Kurzyniec S, Nordblom GD, et al. Glucuronidation of prodrug reactive site: isolation and characterization of oxymethylglucuronide metabolite of fosphenytoin. Clin Chem. 2001;47(5):910-918.

14. Kriel RL, Cifuentes RF. Fosphenytoin in infants of extremely low birth weight. Pediatr Neurol. 2001;24(3):219-221.

15. Pryor FM, Gidal B, Ramsay RE, et al. Fosphenytoin: pharmacokinetics and tolerance of intramuscular loading doses. Epilepsia. 2001; 42(2):245-250.

16. Touchette DR, Rhoney DH. Cost-minimization analysis of phenytoin and fosphenytoin in the emergency department. Pharmacotherapy. 2000;20(8):908-916.

17. De Toledo JC, Ramsay RE. Fosphenytoin and phenytoin in patients with status epilepticus: improved tolerability versus increased costs. Drug Saf. 2000;22(6):459-466.

18. Armstrong EP, Sauer KA, Downey MJ. Phenytoin and fosphenytoin: a model of cost and clinical outcomes. Pharmacotherapy. 1999; 19(7):844-853.

19. Chaney LA, Rockhold RW, Wineman RW, et al. Anticonvulsant-resistant seizures following pyridostigmine bromide (PB) and N,N-diethyl-mtoluamide (DEET). Toxicol Sci. 1999;49(2):306-311.
20. Chan SA, Reid KH, Schurr A, et al. Fosphenytoin reduces hippocampal neuronal damage in rat following transient global ischemia. Acta Neurochir (Wien). 1998;140(2):175-180.

21. Aweeka FT, Gottwald MD, Gambertoglio JG. Pharmacokinetics of fosphenytoin in patients with hepatic or renal disease. Epilepsia. 1999;40(6):777-782.

22. Burstein AH, Cox DS, Mistry B, et al. Phenytoin pharmacokinetics following oral administration of phenytoin suspension and fosphenytoin solution to rats. Epilepsy Res. 1999;34(3):129-133.

23. Dasgupta A, Warner BF, Datta P. Use of alkaline phosphatase to correct the underestimation of fosphenytoin concentration in serum measured by phenytoin immunoassays. Am J Clin Pathol. 1999;111(4):557-562.

24. Walton NY, Uthman BM, Yafi El, et al. Phenytoin penetration into brain after administration of phenytoin or fosphenytoin. Epilepsia. 1999;40(2):153-156.

25. Takeoka M, Krishnamoorthy KS, Soman TB, et al. Fosphenytoin in infants. J Child Neurol. 1998;13(11):537-540.

26. Dasgupta A, Havlik D. Elevated free fosphenytoin concentrations in uremic sera: uremic toxins hippuric acid and indoxyl sulfate do not account for the impaired protein binding of fosphenytoin. Ther Drug Monit. 1998;20(6):658-662.

27. Ramsay RE, Wilder BJ, Uthman BM, et al. Intramuscular fosphenytoin (Cerebyx) in patients requiring a loading dose of phenytoin. Epilepsy Res. 1997;28(3):181-187.

28. Kugler AR, Annesley TM, Nordblom GD, et al. Cross-reactivity of fosphenytoin in two human plasma phenytoin immunoassays. Clin Chem. 1998;44(7):1474-1480.

29. Löscher W, Reissmüller E, Ebert U. Anticonvulsant effect of fosphenytoin in amygdala-kindled rats: comparison with phenytoin. Epilepsy Res. 1998;30(1):69-76.

30. Luer MS. Fosphenytoin. Neurol Res. 1998;20(2):178-182.

31. Knapp LE, Kugler AR. Clinical experience with fosphenytoin in adults: pharmacokinetics, safety, and efficacy. J Child Neurol. 1998; 13(1):15-18.

32. Brown TR. Fosphenytoin (Cerebyx). Clin Neuropharmacol. 1997;20(1):1-12.

33. Lai CM, Moore P, Quon CY. Binding of fosphenytoin, phosphate ester pro drug of phenytoin, to human serum proteins and competitive binding with carbamazepine, diazepam, phenobarbital, phenylbutazone, phenytoin, valproic acid or warfarin. Res Commun Mol Pathol Pharmacol. 1995;88(1):51-62.

34. Jamerson BD, Dukes GE, Brouwer KL, et al. Venous irritation related to intravenous administration of phenytoin versus fosphenytoin. Pharmacotherapy. 1994;14(1):47-52.

35. Fierro LS, Savulich DH, Benezra DA. Safety of fosphenytoin sodium. Am J Health Syst Pharm. 1996;53(22):2707-2712.

36. Boucher BA. Fosphenytoin: a novel phenytoin prodrug. Pharmacotherapy. 1996;16(5):777-791

37. Wilder BJ, Campbell K, Ramsay RE, et al. Safety and tolerance of multiple doses of intramuscular fosphenytoin substituted for oral phenytoin in epilepsy or neurosurgery. Arch Neurol. 1996;53(8):764-768.

38. Boucher BA, Feler CA, Dean JC, et al. The safety, tolerability, and pharmacokinetics of fosphenytoin after intramuscular and intravenous administration in neurosurgery patients. Pharmacotherapy. 1996;16(4):638-645. 
39. Browne TR, Kugler AR, Eldon MA. Pharmacology and pharmacokinetics of fosphenytoin. Neurology. 1996;46(6):3-7.

40. Uthman BM, Wilder BJ, Ramsay RE. Intramuscular use of fosphenytoin an overview. Neurology. 1996;46(6):24-28.

41. Ramsay RE, De Toledo J. Intravenous administration of fosphenytoin options for the management of seizures. Neurology. 1996;46(6):17-19.

42. Paloucek FP. Fosphenytoin safety and economics. Am J Health Syst Pharm. 1996;53(22):2702.

43. Bebin M, Bleck TP. New anticonvulsant drugs. Focus on flunarizine, fosphenytoin, midazolam and stiripentol. Drugs. 1994;48(2):153-171.

44. Koul R, Deleu D. Subtherapeutic free phenytoin levels following fosphenytoin therapy in status epilepticus. Neurology. 2002;58(1):147-148.

45. Labiner DM. Data vs opinion, phenytoin vs fosphenytoin: the saga continues. Arch Intern Med. 1999;159(22):2631-2632.

46. Fierro L, Hudak J. Cost of fosphenytoin. Ann Emerg Med. 1998;31(1):137-138.

47. Bradbury-Golas K, Carson L. Fosphenytoin: a feasible alternative to phenytoin for seizure therapy. J Emerg Nurs. 1996;22(5):431-432.

48. Ten Hoor CN, Stewart BH. Reconversion of fosphenytoin in the presence of intestinal alkaline phosphatase. Pharm Res. 1995;12(11):1806-1809.

49. Bosch J, Roca T, Domènech J, et al. Synthesis of water-soluble phenytoin prodrugs. Bioorg Med Chem Lett. 1999;9(13):1859-1862.

50. Hussey EK, Dukes GE, Messenheimer JA, et al. Evaluation of the pharmacokinetic interaction between diazepam and ACC-9653 (a phenytoin prodrug) in healthy male volunteers. Pharm Res. 1990;7(11):1172-1176.

51. Jamerson BD, Donn KH, Dukes GE, et al. Absolute bioavailability of phenytoin after 3-phosphoryloxymethyl phenytoin disodium (ACC-9653) administration to humans. Epilepsia. 1990;31(5):592-597.

52. Walton NY, Treiman DM. Efficacy of ACC-9653 (a phenytoin prodrug) in experimental status epilepticus in the rat. Epilepsy Res. 1990;5(2):165-168.

53. Boucher BA, Bombassaro AM, Rasmussen SN, et al. Phenytoin prodrug 3-phosphoryloxymethyl phenytoin (ACC-9653): pharmacokinetics in patients following intravenous and intramuscular administration. $J$ Pharm Sci. 1989;78(11):929-932.
54. Smith RD, Brown BS, Maher RW, et al. Pharmacology of ACC-9653 (phenytoin prodrug). Epilepsia.1989;30(2):15-21.

55. Browne TR, Davoudi H, Donn KH, et al. Bioavailability of ACC-9653 (phenytoin prodrug). Epilepsia. 1989;30(2):27-32.

56. Leppik IE, Boucher R, Wilder BJ, et al. Phenytoin prodrug: preclinical and clinical studies. Epilepsia. 1989;30(2):22-26.

57. Gerber N, Mays DC, Donn KH, et al. Safety, tolerance and pharmacokinetics of intravenous doses of the phosphate ester of 3-hydroxymethyl-5,5diphenylhydantoin: a new prodrug of phenytoin. $J$ Clin Pharmacol. 1988;28(11):1023-1032.

58. Holliday SM, Benfield P, Plosker GL. Fosphenytoin. Pharmacoeconomic implications of therapy. Pharmacoeconomics. 1998;14(6):685-690.

59. Bialer M. New antiepileptic drugs that are second generation to existing antiepileptic drugs. Expert Opin Investig Drugs. 2006;15(6):637-647.

60. Chisholm D. Cost-effectiveness of first-line antiepileptic drug treatments in the developing world: a population-level analysis. Epilepsia. 2005;(46):751-759.

61. Dwivedi C. Antiepileptic Drugs. Am J Pharm Educ. 2001;(65):197-202.

62. Gerlach AC, Krajewski JL. Antiepileptic Drug Discovery and Development: What Have We Learned and Where Are We Going? Pharmaceuticals. 2010;(3):2884-2899.

63. Kwan P, Sander JW. The natural history of epilepsy: An epidemiological view. J Neurol Neurosurg Psychiatry. 2004;(75):1376-1381.

64. Macdonald RL, Greenfield LJ. Mechanisms of action of new antiepileptic drugs. Curr Opin Neurol. 1997;(10):121-128.

65. Rogawski MA, Loscher W. The neurobiology of antiepileptic drugs. Nat Rev Neurosci. 2004;(5):553-564.

66. Wagh RV, Antre RV, Oswal RJ, et al. Anticonvulsant Activity: An Overview. J Pharm Res \& Bio Scientific Res. 2011;1(3):142-147. 